\title{
A stabilizing MPC algorithm using perfomance bounds from saturated linear feedback
}

\author{
Johan Löfberg \\ Division of Automatic Control Department of Electrical \\ Engineering \\ Linköpings universitet, SE-581 83 Linköping, Sweden \\ WWW: http://Www. control.isy.liu.se \\ Email: johanl@isy.liu.se
}

January 8, 2001

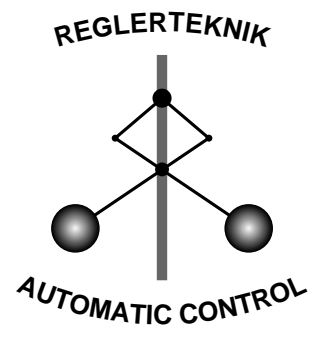

LINKÖPING

Report No.: LiTH-ISY-R-2327

Submitted to CDC2000

Technical reports from the Automatic Control group in Linköping are available by anonymous ftp at the address ftp.control.isy.liu.se. This report is contained in the file 2327 .pdf. 


\begin{abstract}
We present a method to increase feasibility in MPC algorithms that use ellipsoidal terminal state constraints and performance bounds from nominal controllers. The method is based on estimating a bound on the achievable performance with a saturated nominal controller and using this bound in the MPC algorithm. The resulting MPC controller can be implemented efficiently with Second Order Cone Programming.
\end{abstract}

\title{
Keywords: MPC, Stability, LMI
}




\title{
A stabilizing MPC algorithm using performance bounds from saturated linear feedback
}

\author{
Johan Löfberg \\ Department of Electrical Engineering \\ Linköping University, SE-581 83 Linköping, Sweden \\ WWW: http://www. control.isy.liu.se \\ Email: johanl@isy.liu.se
}

\begin{abstract}
We present a method to increase feasibility in MPC algorithms that use ellipsoidal terminal state constraints and performance bounds from nominal controllers. The method is based on estimating a bound on the achievable performance with a saturated nominal controller and using this bound in the MPC algorithm. The resulting MPC controller can be implemented efficiently with Second Order Cone Programming.
\end{abstract}

\section{Introduction}

Many proposed MPC algorithms are based on incorporating an upper bound of the achievable performance, or cost, with a nominal unsaturated controller, into the original optimization problem. In addition, an invariant domain where this bound holds is added to the optimization problem as a terminal state constraint. Common choices of such terminal constraints are ellipsoidal and zero state constraints, i.e. constraints in the form $x(k+N)^{T} P x(k+N) \leq 1$. By using such strategies, stability can be guaranteed if the optimization problem is feasible in the initial state, see [8] for a recent survey on the topic. Due to the added terminal state constraint, feasibility might however be a problem.

In this report we extend these ideas in order to create less conservative terminal state constraints, thus making the optimization feasible for a larger initial state domain. The idea is to calculate an upper bound of the achievable performance, when using a saturated controller, and an invariant ellipsoidal domain where this bound holds.

In section 2 and 3, we introduce some needed background and describe a model of the saturation. Using this model, we derive an upper bound of the achievable performance in section (5). This bound is improved upon in section (6), and in the last sections, we show how this improved bound can be used in MPC.

\section{Preliminaries}

We consider discrete-time systems

$$
x(k+1)=A x(k)+B u(k)
$$

with $x(k) \in \mathbf{R}^{n}$ and $u(k) \in \mathbf{R}^{m}$. The system is subjected to (normalized) control constraints, $\left|u_{i}(k)\right| \leq 1$.

A lot of analysis will be based on invariant ellipsoids

Definition 1 (Ellipsoid) For $W=W^{T} \succ 0$, an ellipsoidal set is defined as

$$
\mathcal{E}_{W}=\left\{x \mid x^{T} W x \leq 1\right\}
$$

An important fact used in this work is that the volume of the ellipsoid is proportional to $\sqrt{\operatorname{det}\left(W^{-1}\right)}$.

When we work with LMIs, the following result will be used repeatedly.

Lemma 1 (Schur complement) Equivalence holds between the two conditions

$$
X \succ 0, X-Z W^{-1} Z^{T} \succ 0 \Leftrightarrow\left[\begin{array}{cc}
X & Z \\
Z^{T} & W
\end{array}\right] \succ 0
$$

Proof: See [2].

The importance of this Lemma is that nonlinear matrix inequalities can be expressed as equivalent linear inequalities.

\section{Saturation modeling}

The algorithm in this work is based on a polytopic representation of the saturated system. Polytopic representations are simple means to conservatively model nonlinearities as uncertainties in a linear system, see [2]. Using a polytopic uncertainty model to analyze 
stability of a saturated system is a standard procedure, and has been done before, see, e.g, [1]. Before we proceed with the description of the polytopic model, we introduce the concept saturation level.

Definition 2 (Saturation level) For a system with control constraints, $\left|u_{i}(k)\right| \leq 1$, we define the saturation levels as

$$
\gamma_{i}(k)= \begin{cases}1 & \left|u_{i}(k)\right| \leq 1 \\ \frac{1}{\left|u_{i}(k)\right|} & \left|u_{i}(k)\right|>1\end{cases}
$$

By introducing

$$
\Gamma(k)=\operatorname{diag}\left(\gamma_{1}(k), \ldots, \gamma_{m}(k)\right)
$$

the saturated control can be written as $\Gamma(k) u(k)$

If we know that the control $u_{i}$ never saturates to a level less than $\gamma_{\min , i}$, i.e.

$$
\gamma_{\min , i} \leq \gamma_{i}(k) \leq 1
$$

we can (conservatively) describe $\Gamma(k)$ as an interpolation of the combinatorial combination of the diagonal matrices $\Gamma_{j}$ having elements either 1 or $\gamma_{\min , i}$

$$
\Gamma(k)=\sum_{j=1}^{2^{m}} \lambda_{j} \Gamma_{j} \quad \sum_{1}^{2^{m}} \lambda_{j}=1, \lambda_{j} \geq 0
$$

We call the extreme values $\Gamma_{j}$ the corners of the polytopic description.

\section{Domain of attraction for saturated system}

The first thing we do is to find an invariant domain in the state space where the saturated system is stable. This problem has been addressed recently by several authors, see $[4,1,3]$ just to mention a few.

The perhaps most simple method is to search for a Lyapunov function $x^{T} W x$ and an invariant domain $\mathcal{E}_{W}$. For an unstable system, $\mathcal{E}_{W}$ not can be made arbitrarily large, since it is not possible to globally stabilize an unstable system subject to control constraints. Given allowed saturation levels $\gamma_{\min , i}$, the problem is therefore to find the largest invariant set $\mathcal{E}_{W}$ that lies in the set where $\gamma_{i} \geq \gamma_{\min , i}$. We assume that the controller before saturation is $u=-L x, L=\left[\begin{array}{llll}l_{1}^{T} & l_{2}^{T} & \cdots & l_{m}^{T}\end{array}\right]^{T}$.

A solution to this problem is easily derived and leads to the following optimization problem

$$
\begin{aligned}
\text { maximize } \operatorname{det}\left(W^{-1}\right) & \\
(A-B \Gamma(k) L)^{T} W(A-B \Gamma(k) L)-W & \prec-\epsilon W \\
l_{i} W^{-1} l_{i}^{T} & \leq\left(\frac{1}{\gamma_{i}}\right)^{2}
\end{aligned}
$$

The maximization comes from the volume definition of an ellipsoid, which we wish to maximize. The first inequality assures $x^{T} W x$ to be a Lyapunov function, with a decay-rate $\epsilon>0$. The last inequality is based on the fact that $\max _{x \in \mathcal{E}_{W}} a^{T} x=\sqrt{a^{T} W^{-1} a}$ [2], i.e. we allow a saturation $\gamma_{i}$. By pre-and post-multiplying (1) with $W^{-1}$ and applying a Schur complement, the equations above can written in as a LMI if we introduce $Y=W^{-1}$. Due to the second inequality, we know that $\gamma_{i}(k) \geq \gamma_{\min , i}$, so the polytopic description of $\Gamma(k)$ described in section 3 can be used, hence we replace $\Gamma(k)$ by its vertices $\Gamma_{j}$. All together, we obtain the following sufficient LMI, a MAXDET [9] problem.

$$
\begin{aligned}
\text { maximize } \operatorname{det}(Y) & \\
{\left[\begin{array}{cc}
(1-\epsilon) Y & Y\left(A-B \Gamma_{j} L\right)^{T} \\
\left(A-B \Gamma_{j} L\right) Y & Y
\end{array}\right] } & \succ 0 \\
l_{i} Y l_{i}^{T} & \leq\left(\frac{1}{\gamma_{\text {min }, i}}\right)^{2}
\end{aligned}
$$

To be precise, the expression above is a BMI, since $\gamma_{\text {min, } i}$ are free variables. For fixed $\gamma_{\text {min,i } i}$ the problem is however a LMI. The joint optimization can be solved (local minima) with various techniques, typically using some alternating methods or linearization scheme. See [3] for one approach. For $m \leq 2$, the problem is most easily solved by gridding. We will not elaborate on these issues in this work, so we assume the optimization problem can be solved reasonably well.

By solving the optimization problem, we get an estimation of the domain where a saturated controller is guaranteed to stabilize the system. Our next step is to estimate the achievable performance in this domain.

\section{A quadratic bound of achievable cost}

Our goal is to find an upper bound of the cost

$J(x(k))=\sum_{i=k}^{\infty} x^{T}(i) Q x(i)+u^{T}(i) R u(i) \quad Q, R \succ 0$

by finding a matrix $S=S^{T} \succ 0$ such that

$$
J(x(k)) \leq x^{T}(k) S x(k)
$$

It is easy to see that the following growth condition will guarantee the upper bound to hold

$$
\begin{aligned}
x^{T}(k+1) S x(k+1) & -x^{T}(k) S x(k) \leq \\
& -x^{T}(k) Q x(k)-u^{T}(k) R u(k)
\end{aligned}
$$

By inserting the dynamics $x(k+1)=A x(k)+B u(k)$ and the saturated controller $u(k)=-\Gamma(k) L x(k)$ we have the matrix inequality

$$
\begin{aligned}
(A-B \Gamma(k) L)^{T} S(A-B \Gamma(k) L)-S \prec \\
-Q-L^{T} \Gamma(k)^{T} R \Gamma(k) L
\end{aligned}
$$


Pre- and post-multiply (2) with $Y=S^{-1}$, apply a Schur complement and use the polytopic description of $\Gamma(k)$, and we obtain

$$
\left[\begin{array}{cccc}
Y & Y\left(A-B \Gamma_{j} L\right)^{T} & Y & Y L^{T} \Gamma_{j}^{T} \\
\left(A-B \Gamma_{j}\right) Y & Y & 0 & 0 \\
Y & 0 & Q^{-1} & 0 \\
\Gamma_{j} L Y & 0 & 0 & R^{-1}
\end{array}\right] \succ 0
$$

For notational purposes, we prefer to go back to the original form

$$
\left(A-B \Gamma_{j} L\right)^{T} S\left(A-B \Gamma_{j} L\right)-S \prec-Q-L^{T} \Gamma_{j}^{T} R \Gamma_{j} L
$$

Our goal is to create a small bound $x^{T}(k) S x(k)$. This is equivalent to making $S$ small. To do this, we minimize the largest eigenvalue of $S$

Theorem 1 (Quadratic upper bound) An upper bound of the cost (1) for $x(k) \in \mathcal{E}_{W}$ is

$$
J(x(k)) \leq x^{T}(k) S x(k)
$$

where $S$ is given as the solution to

$$
\begin{aligned}
\text { minimize } t & \\
\left(A-B \Gamma_{j} L\right)^{T} S\left(A-B \Gamma_{j} L\right)-S & \prec-Q-L^{T} \Gamma_{j}^{T} R \Gamma_{j} L \\
S & \prec t I
\end{aligned}
$$

Proof: By construction above.

\section{An improved piecewise quadratic bound}

The upper bound we have derived is in general very conservative. The reason is that if we have chosen saturation levels such that the system is very close to unstable in some parts, the upper bound has to take these domains with extremely slow convergence into account.

The bound holds in the whole set $\mathcal{E}_{W}$, but it is clear that we can create a better bound in smaller sets contained in $\mathcal{E}_{W}$. We will therefore formulate a less conservative bound that is based on a piecewise quadratic function in a special form.

$$
J \leq \max _{i}\left(x^{T} S_{i} x-\rho_{i}\right)
$$

The reason we are using this kind of function is that it is easy and efficient to use in a MPC application.

Remark: It should be pointed out that piecewise quadratic functions of more general structure are available in the literature, see e.g [5]. Using those methods to estimate the cost would most likely give better bounds, but they do not lend them selves to be incorporated in a convex MPC algorithm.
The derivation of the upper bound is a bit messy, so we start by presenting the result

\section{Theorem 2 (Piecewise quadratic upper bound)} An upper bound of the cost (1) for $x(k) \in \mathcal{E}_{W}$ is

$$
J(x(k)) \leq \max _{i}\left(x^{T}(k) S_{i} x(k)-\rho_{i}\right)
$$

where $S_{i}$ and $\rho_{i}$ are calculated with

$$
\begin{gathered}
\text { minimize } t \\
\begin{array}{c}
\left(A-B \Gamma_{j i} L\right)^{T} S_{i}\left(A-B \Gamma_{j i} L\right)-S_{i} \prec-Q-L^{T} \Gamma_{j i}^{T} R \Gamma_{j i} L \\
\left(\rho_{l+1}-\rho_{l}\right) I \prec \beta_{l}^{-1} W^{-1 / 2}\left(S_{l+1}-S_{l}\right) W^{-1 / 2} \\
W^{-1 / 2} S_{n_{s}} W^{-1 / 2} \prec\left(t+\rho_{n_{s}}\right) I \\
\rho_{1}=0 \\
j=1 \ldots 2^{m}, i=1 \ldots n_{s}, l=1 \ldots n_{s}-1
\end{array}
\end{gathered}
$$

The variables are defined in the derivation below.

The idea is to create a number of smaller sets $\mathcal{E}_{\beta W}$. $\beta \geq 1$ is a parameter that scales the ellipsoid, see the definition in section 1 . $\beta=1$ corresponds to the original ellipsoid, and $\beta=\max _{i}\left(l_{i} W^{-1} l_{i}^{T}\right)$ corresponds to the case when the ellipsoid is scaled so that, for all $i, \gamma_{i}=1$ in $\mathcal{E}_{\beta W}$, i.e. no saturation occurs.

We define $n_{s} \geq 2$ ellipsoids

$$
\mathcal{E}_{\beta_{i} W} \quad i=1 \ldots n_{s}
$$

and their corresponding scalings

$$
\begin{aligned}
\beta_{1} & =\max _{i}\left(L_{i} W^{-1} L_{i}^{T}\right) \quad i=1 \ldots m \\
\beta_{i+1} & <\beta_{i} \quad 1 \leq i \leq n_{s}-1 \\
\beta_{n_{s}} & =1
\end{aligned}
$$

In each of these ellipsoids, the growth condition (2) must hold. The difference in the different ellipsoids is the saturation levels. We therefore have to calculate the corners $\Gamma_{j i}$, where index $i$ represents the different ellipsoids and $j$ represents the corners in the description, in each ellipsoid. This leads to the first inequality in Theorem 2. This LMI will give us the bound $J(x) \leq x^{T} S_{i} x$ when $x \in \mathcal{E}_{\beta_{i} W}$. An important fact is that the condition (2) we have used to derive $S_{i}$ only concerns growth conditions. The idea is therefore to subtract a constant positive scalar, $\rho_{i}$. If we want our bound to be as low as possible, our goal is to make $\rho_{i}$ as large as possible. In the inner most domain, the bound $x^{T} S_{1} x-\rho_{1}$ will be used. Since the cost in the origin equals zero, we must have $\rho_{1}=0$. What about $\rho_{2}$ ? As soon as $x \in \mathcal{E}_{\beta_{2} W} \backslash \mathcal{E}_{\beta_{1} W}$ (outside the innermost ellipsoid but still in the second ellipsoid), the upper bound should use $x^{T} S_{2} x-\rho_{2}$. This will be the case if

$$
x^{T} S_{2} x-\rho_{2} \geq x^{T} S_{1} x-\rho_{1} \quad \text { when } x^{T} \beta_{1} W x=1
$$


or, equivalently

$$
x^{T}\left(S_{2}-S_{1}\right) x \geq \rho_{2}-\rho_{1} \quad \text { when } x^{T} \beta_{1} W x=1
$$

The following lemma will be useful

Lemma 2 (Max and min of $x^{T} S x$ when $x^{T} W x=1$ ) The maximum and minimum of $x^{T} S x$ on the set $x^{T} W x=1\left(S=S^{T} \succ 0, W=W^{T} \succ 0\right)$ is given by

$$
\begin{aligned}
\max x^{T} S x & =\lambda_{\max }\left(W^{-1 / 2} S W^{-1 / 2}\right) \\
\min x^{T} S x & =\lambda_{\min }\left(W^{-1 / 2} S W^{-1 / 2}\right)
\end{aligned}
$$

Proof: Introduce $z=W^{1 / 2} x$, and the problem is to maximize (minimize) $z^{T} W^{-1 / 2} S W^{-1 / 2} z$ when $z^{T} z=1$. The optimal choice is to pick $z$ to be the normalized eigenvector corresponding to the largest (smallest) eigenvalue, and the lemma follows directly.

By looking at the minima of the left hand side of (3), the lemma above gives us (after pulling out $\beta_{1}$ )

$$
\beta_{1}^{-1} \lambda_{\min }\left(W^{-1 / 2}\left(S_{2}-S_{1}\right) W^{-1 / 2}\right) \geq \rho_{2}-\rho_{1}
$$

The same argument holds in the general case and results in the constraint

$\beta_{i-1}^{-1} \lambda_{\min }\left(W^{-1 / 2}\left(S_{i}-S_{i-1}\right) W^{-1 / 2}\right) \geq \rho_{i}-\rho_{i-1}$

This is a linear convex constraint, equivalent to the second inequality in Theorem 2 .

So far we have only introduced inequalities, but nothing to optimize. There are probably many choices, but one suitable term to minimize could be the worst case upper bound on the border of $\mathcal{E}_{W}$

$\operatorname{minimize}\left(\max x^{T} S_{n_{s}} x-\rho_{n_{s}} \quad\right.$ when $\left.x^{T} W x=1\right)$

Once again we use the lemma above and formulate this as $\left(\beta_{n_{s}}=1\right)$

$$
\text { minimize } \lambda_{\max }\left(W^{-1 / 2} S_{n_{s}} W^{-1 / 2}\right)-\rho_{n_{s}}
$$

This gives us the last inequality in the theorem.

To see the benefits of using this more complicated bound, we give an example

Example 1 (Improved upper bound) We have the following set-up (discretized double integrator)

$$
A=\left[\begin{array}{ll}
1 & 0 \\
1 & 1
\end{array}\right], B=\left[\begin{array}{c}
1 \\
0.5
\end{array}\right], Q=\left[\begin{array}{ll}
1 & 0 \\
0 & 1
\end{array}\right], R=1
$$

$L$ is designed as the corresponding LQ state feedback. Stability can be proven for $\gamma_{\min } \geq 0.24$ and we find

$$
W=\left[\begin{array}{cc}
0.079 & 0.017 \\
0.017 & 0.0155
\end{array}\right]
$$

With the simple upper bound $x^{T} S x$ we get

$$
S=\left[\begin{array}{cc}
452 & 88 \\
88 & 94
\end{array}\right]
$$

and the worst case upper bound on (1) when $x(k) \in \mathcal{E}_{W}$ is 6554. We now chose to have three ellipsoids, $n_{s}=3$, in order to create a piecewise quadratic upper bound. $\beta_{1}$ and $\beta_{3}$ are defined according to earlier, and $\beta_{2}$ was $=0.9^{-2}$. This means that the middle ellipsoid $\mathcal{E}_{\beta_{2} W}$ has a radius 0.9 times the radius of the largest ellipsoid $\mathcal{E}_{\beta_{3} W}$. Solving the optimization problem yields

$$
\begin{aligned}
S_{1} & =\left[\begin{array}{ll}
2.59 & 1.12 \\
1.12 & 2.37
\end{array}\right], S_{2}=\left[\begin{array}{cc}
24.9 & 5.8 \\
5.8 & 6.8
\end{array}\right], S_{3}=\left[\begin{array}{cc}
483 & 97 \\
97 & 100
\end{array}\right] \\
\rho_{1} & =0, \rho_{2}=16.3, \rho_{3}=4614
\end{aligned}
$$

The worst case cost is now 2094. Even more important to note is that the optimal cost for the unsaturated system, i.e. standard $L Q$ control, is

$$
J_{L Q}(x(k))=x^{T}(k)\left[\begin{array}{ll}
2.59 & 1.12 \\
1.12 & 2.37
\end{array}\right] x(k)
$$

Close to the origin, our upper bound will be $x^{T}(k) S_{1} x(k)$, i.e. the bound is tight there. This should be compared to the simple quadratic bound which will overestimate the unsaturated cost by a factor $\sim 100$.

\section{Increasing feasibility in MPC}

The motivation for this work has has been to increase feasibility in some MPC algorithms with terminal constraints. The problem that occurs when terminal state constraints are used, is that the terminal domain might not be in the reachable set from the initial condition, due to the constrained control. However, compared to early approaches where the terminal domain was the origin, i.e the constraint $x(k+N)=0$ was used, an ellipsoidal domain is clearly a step forward. The standard approach to find a terminal domain is to pick a nominal linear feedback, $u=-L x$, and find an invariant domain where the nominal feedback not saturates. This domain is used for the terminal constraint. Once again, see [8] for a survey on related schemes. The contribution in this work is that since we not assume the nominal controller to be unsaturated, we are able to derive larger terminal state domains for a given $L$. With the larger terminal domain, the initial feasible domain will be larger.

For simple notation, we define the derived upper bound

$$
\Phi(x)=\max _{i} x^{T} S_{i} x-\rho_{i} \quad i=1 \ldots n_{s}
$$

and the cost to minimize

$$
\begin{aligned}
J(k)=\sum_{j=k}^{k+N-1}\left[x^{T}(j) Q x(j)+u^{T}(j)\right. & R u(j)] \\
& +\Phi(x(k+N))
\end{aligned}
$$


Theorem 3 (Stability of MPC algorithm) The

$M P C$ controller is defined by calculating $u(k)$ with the following optimization

$$
\begin{aligned}
\operatorname{minimize} J(k) & \\
\left|u_{i}(j)\right| & \leq 1 \\
x(k+N) & \in \mathcal{E}_{W}
\end{aligned}
$$

Stability of the closed loop is guaranteed if the problem is feasible at in the initial state at time $k=0$.

Proof: We follow the standard approach and use the optimal cost $J^{*}(k)$ as a Lyapunov function. To begin with, we study $J(k+1)$ with an arbitrary choice of $u$. We add and subtract some terms in order to get an expression containing $J(k)$

$$
\begin{gathered}
J(k+1)=\sum_{j=k+1}^{k+N}\left[x^{T}(j) Q x(j)+u^{T}(j) R u(j)\right] \\
\quad+\Phi(x(k+N+1)) \\
=\sum_{j=k}^{k+N-1}\left[x^{T}(j) Q x(j)+u^{T}(j) R u(j)\right] \\
+\Phi(x(k+N))+\Phi(x(k+N+1)))-\Phi(x(k+N)) \\
\quad-x^{T}(k) Q x(k)-u^{T}(k) R u(k) \\
+x^{T}(k+N) Q x(k+N)+u^{T}(k+N) R u(k+N)
\end{gathered}
$$

We denote the optimal solution at time $k u^{*}$. Since $u$ is arbitrary in the expression above, we can pick $u^{*}$ the first $N$ samples. With this choice of $u$, we see that the first two terms define the previously optimal cost

$$
\begin{gathered}
J(k+1)=J^{*}(k)-x^{T}(k) Q x(k)-u^{* T}(k) R u^{*}(k) \\
+\Phi(x(k+N+1))-\Phi(x(k+N)) \\
+x^{T}(k+N) Q x(k+N)+u^{T}(k+N) R u(k+N)
\end{gathered}
$$

We define the increment in the terminal state cost in order to be able to bound it from above

$$
\Delta=\Phi(x(k+N+1))-\Phi(x(k+N))
$$

By using the definition of $\Phi$, we write $\Delta$ as

$$
\begin{aligned}
\Delta=\max _{i}\left[x^{T}(k+N+1) S_{i} x(k+N+1)-\rho_{i}\right] \\
-\max _{i}\left[x^{T}(k+N) S_{i} x(k+N)-\rho_{i}\right]
\end{aligned}
$$

At time $k$, the optimal solution fulfills the terminal state constraint, i.e. $x(k+N) \in \mathcal{E}_{W}$. Since $\mathcal{E}_{W}$ is invariant with $u(k+N)=-\Gamma(k+N) L x(k+N)$, this is a feasible extension of the old solution, since terminal and control constraint will be fulfilled.

Inserting $x(k+N+1)=(A-B \Gamma(k+N) L) x(k+N)$, and using the first inequality in Theorem 2 , we obtain

$\Delta \leq-x^{T}(k+N)(Q+L \Gamma(k+N) R \Gamma(k+N) L) x(k+N)$
If we insert this into (4) together with the choice of $u(k+N)$, we see that the sum of the two last rows in (4) is negative. Hence,

$$
J(k+1) \leq J^{*}(k)-x^{T}(k) Q x(k)-u^{T}(k) R u(k)
$$

Since the control signal we have used in the proof not necessarily is optimal, we have $J^{*}(k+1) \leq J(k+1)$. This leads us to the final inequality

$$
J^{*}(k+1) \leq J^{*}(k)-x^{T}(k) Q x(k)-u^{T}(k) R u(k)
$$

Hence, $J^{*}(k)$ proves asymptotic stability.

\subsection{SOCP formulation of MPC algorithm}

The max function can be efficiently implemented in the optimization since it can be written as a second order cone constraint [7]. A second order cone constraint is a special case LMI for which specially purpose solvers are available. By defining the vectors with future $u$ and predicted states

$$
X=\left[\begin{array}{c}
x(k) \\
x(k+1) \\
\vdots \\
x(k+N-1)
\end{array}\right], \quad U=\left[\begin{array}{c}
u(k) \\
u(k+1) \\
\vdots \\
u(k+N-1)
\end{array}\right]
$$

the predicted states can be written as

$$
X=F+G U
$$

where $F \in \mathbf{R}^{N n \times 1}$ and $G \in \mathbf{R}^{N n \times N m}$ are defined in the usual manner. Define $\bar{Q}=\operatorname{diag}(Q, Q, \ldots, Q) \in$ $\mathbf{R}^{N n \times N n}$ and $\bar{R}=\operatorname{diag}(R, R, \ldots, R) \in \mathbf{R}^{N m \times N m}$. The MPC algorithm in Theorem 3 is solved with the following SOCP

$$
\begin{aligned}
\text { minimize } s+t & \\
(F+G U)^{T} \bar{Q}(F+G U)+U^{T} \bar{R} U & <t \\
\left|u_{i}(j)\right| & \leq 1 \\
x^{T}(k+N) S_{i} x(k+N)-\rho_{i} & \leq s \\
x^{T}(k+N) W x(k+N) & \leq 1
\end{aligned}
$$

The difference compared to a standard MPC algorithm is the two last quadratic inequalities, which force us to use SOCP instead of QP. Note that this always is needed when ellipsoidal terminal state constraints are used, and is not due to the extensions in this paper.

\subsection{Simulation results}

We conclude this paper with an example to show the resulting closed loop behavior of the proposed MPC algorithm.

Example 2 (MPC) We apply the MPC algorithm to the sampled double integrator from the previous example. The parameters used are the same as in Example, except $L=\left[\begin{array}{ll}0.43 & 0.07\end{array}\right]$ (chosen rather arbitrarily 
in order to obtain a large set $\left.\mathcal{E}_{W}\right)$. The prediction horizon was $N=6$. The darker ellipsoid $\mathcal{E}_{W}$ in the figure below is the terminal state ellipsoid, and corresponds to $\gamma_{\min }=0.2$, i.e. we are able to make the terminal domain 5 times larger compared to the unsaturated case. For comparison, we also implemented a standard MPC controller without constraints, using the optimal cost for the unconstrained $L Q$ controller as terminal weight. It is hard to draw any conclusions from

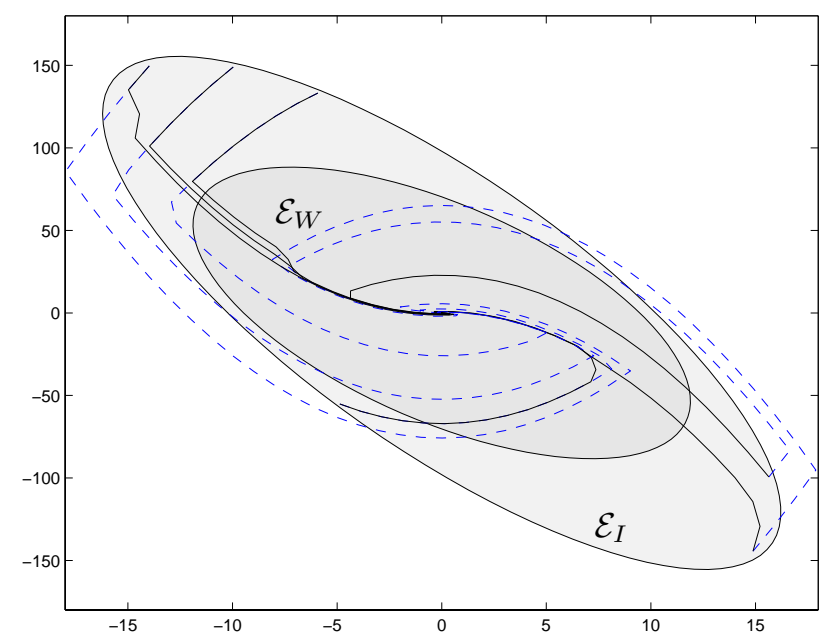

Figure 1: Typical closed loop trajectories. The initial states are chosen to lie close to the boundary of the estimated initial feasible ellipsoid $\mathcal{E}_{I}$, calculated using the results in [6]. Trajectories resulting from the proposed MPC scheme are the solid lines. The dashed lines are the trajectories from the standard MPC algorithm.

the state trajectories shown above. Instead, we study the cost $\sum_{k=0}^{\infty} x^{T}(k) Q x(k)+u^{T}(k) R u(k)$ from different randomly chosen initial states.

\begin{tabular}{|c|c|c|c|}
\hline \multirow{2}{*}{ MPC scheme } & \multicolumn{3}{|c|}{ Cost } \\
\cline { 2 - 4 } & mean & median & max \\
\hline Proposed & $6.7 e 4$ & $7.1 e 3$ & $1.1 e 5$ \\
\hline Standard & $8.8 e 4$ & $9.4 e 3$ & $1.7 e 5$ \\
\hline
\end{tabular}

A slight improvement of performance is achieved. The average improvement is around 20 percent, and up to 60 percent for some initial states. It should be noted is that the main target is guaranteed stability, not improved performance, so the improvement is only a positive sideeffect in this example.

\section{Conclusion}

By using a polytopic representation of a saturated controller, we derived an upper bound on the achievable performance, and an ellipsoidal domain where this bound holds.
The bound was expressed in a special quadratic form that made it possible to be incorporated in a MPC algorithm with ellipsoidal terminal state constraints, leading to a SOCP to be solved on-line. The application of the bound is the main contribution of the presented work, since it gives us the possibility to create softer terminal state constraints. The main drawback of schemes based on terminal constraints is the possible infeasibility, so it is our belief that methods to improve initial feasibility domains are important, since these methods otherwise are hard to justify. We therefor hope that this paper in some sense shows that there are other ways than using unsaturated linear controllers to estimate the upper bound of the achievable cost, a central part in many MPC algorithms with guaranteed stability.

\section{References}

[1] J. Biannic. Stability Analysis of Multi-Variable Feedback Systems with Input Saturations. In $U K A C C$ International Conference on Control 98, 1998.

[2] S. Boyd, L. E. Ghaoui, E. Feron, and V. Balakrishnan. Linear Matrix Inequalities in System and Control Theory. SIAM Studies in Applied Mathematics. SIAM, Philadelphia, Pennsylvania, 1994.

[3] C. Burgat, S. Tarbouriech, and M. Klai. An algorithm for estimating the stability domain of linear discrete-time systems with saturations. In IEEE/SMC Conference, pages 319-324, 1993.

[4] H. Hindi and S. Boyd. Analysis of linear systems with saturation using convex optimization. In Proceedings of 37th Conference on Decision and Control, pages 903-908, 1998.

[5] M. Johansson. Piecewise Linear Control Systems. PhD thesis, Departement of Automatic Control, Lund Institute of Technology, 1999.

[6] Johan Löfberg. Feasibility analysis of MPC with ellipsoidal terminal state constraints. Technical Report LiTH-ISY-R-2216, Department of Electrical Engineering, Linköping University, SE-581 83 Linköping, Sweden, Jun 2000.

[7] M. S. Lobo, L. Vandenberghe, S. Boyd, and H. Lebret. Applications of second-order cone programming. Linear Algebra and its Applications, 284:193-228, 1998.

[8] D.Q. Mayne, J.B. Rawlings, C.V. Rao, and P.O.M. Scokaert. Constrained model predictive control: Stability and optimality. Automatica, 36:789-814, 2000 .

[9] S.P. Wu, L. Vandenberghe, and S. Boyd. MAXDET -Software for Determinant Maximization Problems - User's Guide. Information Systems Laboratory, Electrical Engineering Departement, Stanford University, alpha version edition. 